\title{
Article \\ Bandemia as an Early Predictive Marker of Bacteremia: A Retrospective Cohort Study
}

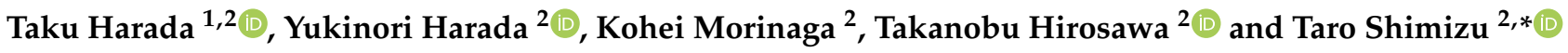 \\ 1 Department of General Medicine, Showa Koto Toyosu University Hospital, Tokyo 135-8577, Japan; \\ hrdtaku@gmail.com \\ 2 Department of Diagnostic and Generalist Medicine, Dokkyo Medical University Hospital, Tochigi 321-0293, \\ Japan; yuki.gym23@gmail.com (Y.H.); k.morinaga0815@gmail.com (K.M.); t.hirosawa1983@gmail.com (T.H.) \\ * Correspondence: shimizutaro7@gmail.com; Tel.: +81-282-86-1111
}

check for updates

Citation: Harada, T.; Harada, Y.; Morinaga, K.; Hirosawa, T.; Shimizu, T. Bandemia as an Early Predictive Marker of Bacteremia: A Retrospective Cohort Study. Int. J. Environ. Res. Public Health 2022, 19, 2275. https://doi.org/10.3390/ ijerph19042275

Academic Editor: Paul B. Tchounwou

Received: 21 December 2021 Accepted: 15 February 2022 Published: 17 February 2022

Publisher's Note: MDPI stays neutral with regard to jurisdictional claims in published maps and institutional affiliations.

Copyright: (C) 2022 by the authors. Licensee MDPI, Basel, Switzerland. This article is an open access article distributed under the terms and conditions of the Creative Commons Attribution (CC BY) license (https:// creativecommons.org/licenses/by/ $4.0 /)$.

\begin{abstract}
This single-center retrospective observational study aimed to verify whether a diagnosis of bandemia could be a predictive marker for bacteremia. We assessed 970 consecutive patients (median age 73 years; male 64.8\%) who underwent two or more sets of blood cultures between April 2015 and March 2016 in both inpatient and outpatient settings. We assessed the value of bandemia (band count $>10 \%$ ) and the percentage band count for predicting bacteremia using logistic regression models. Bandemia was detected in 151 cases $(15.6 \%)$ and bacteremia was detected in 188 cases $(19.4 \%)$. The incidence of bacteremia was significantly higher in cases with bandemia $(52.3 \%$ vs. $13.3 \%$; odds ratio $(\mathrm{OR})=7.15 ; 95 \%$ confidence interval $(\mathrm{CI}) 4.91-10.5)$. The sensitivity and specificity of bandemia for predicting bacteremia were 0.42 and 0.91 , respectively. The bandemia was retained as an independent predictive factor for the multivariable logistic regression model (OR, 6.13; 95\% CI, 4.02-9.40). Bandemia is useful for establishing the risk of bacteremia, regardless of the care setting (inpatient or outpatient), with a demonstrable relationship between increased risk and bacteremia. A bandemia-based electronic alert for blood-culture collection may contribute to the improved diagnosis of bacteremia.
\end{abstract}

Keywords: bandemia; bacteremia; clinical decision support system; blood culture results; bandemiabased electronic alert

\section{Introduction}

Bacteremia is a form of severe infection associated with a high probability of mortality and substantial financial healthcare costs [1].

Bacteremia is a poor prognostic factor for infectious diseases and is one of the leading causes of death in developed countries [2,3]. Specifically, the 1-month rate of communityacquired bacteremia is $10-19 \%$, and the mortality rate for hospital-acquired bloodstream infection is 17-28\% [4-7]. Therefore, the early recognition of bacteremia is clinically important because it could assist in the faster initiation of proper treatment, leading to improved clinical outcomes. In previous studies, the experience of a "chill" has been focused on as a simple and effective predictive factor for bacteremia [8-15], and several prediction rules, which were created by merging multivariate logistic regression models, have been suggested [16-18]. The only single highly predictive factor of bacteremia was a shaking chill, and even then, the prevalence of bacteremia in these cases was only $27 \%$ [1,12]. The clinical rule described by Shapiro et al. is highly sensitive in nature; its external validity has been verified and is useful for preventing the misdiagnosis of bacteremia $[19,20]$. However, its specificity is $29-48 \%$, whilst the prevalence of bacteremia is only $15-37 \%$; besides, the clinical rule requires 11 items of clinical information. Experiencing chills and most of the symptoms described in the existing prediction models are based on patients subjective complaints, all of which require a mastery of interpretation of symptoms, which are highly subjective in nature. Additionally, physicians inaccurately predict-and often 
overestimate-the risk of bacteremia [21]. Therefore, based on certain factors, there is a need for models that can predict the presence of bacteremia, without being influenced by the subjectivity of symptoms that are difficult to accurately assess or may be affected by physician bias.

The clinical decision support system (CDSS) has been demonstrated to improve the quality of medical care in various medical settings; however, its usefulness in diagnosing acute illnesses has not yet been verified [22]. Most of the studies on CDSS in bacteremia were aimed at management and surveillance, and the only report on the prediction of bacteremia by the CDSS is "TREAT" by Paul et al. [23]. TREAT is unique in that it stratified the risk of bacteremia in patients and incorporated 11 different infection sites and 34 different diagnoses, with a $28 \%$ prevalence of bacteremia in the highest risk group. Some studies on machine learning for predicting bacteremia have been conducted; however, the accuracy is still limited [24].

Bandemia is a condition in which $10 \%$ or more counts of band cells (a type of immature neutrophil) are observed in peripheral blood smears, being a potentially useful objective predicting factor for bacteremia [25]. Indeed, the results of studies investigating bandemia as a useful marker for predicting bacteremia, reported in several different settings, have been promising [16,26-29].

Previous CDSS studies on bandemia are unavailable. We hypothesized that a system to generate blood-culture alerts, triggered by new onset bandemia, could contribute to the early diagnosis of bacteremia if incorporated into the electronic medical records; additionally, we tested the association between the presence of bandemia and bacteremia using retrospective data.

\section{Materials and Methods}

\subsection{Patient Selection}

This was a single-center retrospective observational study. We included consecutive outpatients and inpatients who underwent two or more sets of blood cultures and a complete blood count, with a differential white blood cell (WBC) test. Tests were taken $24 \mathrm{~h}$ from the time of the patients' presentation in an outpatient facility and the onset of fever in the inpatient facility at Dokkyo Medical University Hospital between 1 April 2015, and 31 March 2016. The exclusion criteria were developed to exclude factors that can affect WBC differentials or blood culture results and were as follows: age $\leq 14$ years, antibiotic usage the day prior to blood sampling, use of granulocytecolony stimulating factors within 1 week, use of steroids and immunosuppressants within 1 week, chemotherapy for cancer within 4 weeks, history of hematological malignancy or bone metastasis of a solid tumor, irradiation therapy for a malignant tumor, pregnancy, systemic lupus erythematosus, postresuscitation, human immunodeficiency virus infection, or contamination cases. This study was approved by the Ethics Committee of Dokkyo Medical University Hospital and conducted in accordance with the Declaration of Helsinki. The requirement for written informed consent was waived due to the retrospective study design.

\subsection{Data Collections, Outcomes, and Definitions}

Blood culture and WBC differential tests were performed at the discretion of the examining doctors and subsequently performed by an automated analyzer. The sample of the blood cell count were collected by drawing blood into a tube containing an ethylenediaminetetraacetic acid dipotassium. The measurements of the blood cell count, including band count, were analyzed using the Sysmex XN-2000 automatic blood cell analyzer.

We extracted data regarding age, sex, body temperature, WBC count, eosinophil count, percentage band count in the differential WBC test, and blood culture results from the electronic medical records. The primary outcome measurement was bacteremia, which was defined as a positive blood culture 1 week after the samples were collected [20]. We defined contamination as the presence of multiplying coagulase-negative Staphylococcus species, Bacillus species, Propionibacterium acnes, or Corynebacterium species in a single set of 
blood cultures [30]. Bandemia was defined as a band count of $>10 \%$, based on a previous study [8]. Eosinopenia, which has been reported as a risk of bacteremia, was set at a cutoff of 25 cells $/ \mathrm{mm}^{3}$ [31].

\subsection{Statistical Analysis}

Continuous variables are presented as medians, with 25 th and 75 th percentiles, and categorical variables as counts (percentage). Continuous variables were compared using the Wilcoxon rank-sum test. Categorical variables were compared by using chi-square test. Univariate and multivariate logistic regression models were used to assess the predictive values of bandemia. We created two multivariate logistic regression models as follows: the first included age (over 65 or not), sex, body temperature (between 36.0 and 38.0 or not), WBC count (between 4000 and 12,000 or not), and eosinopenia as variables (baseline model); the second added bandemia as a variable to the baseline model (with band model). Furthermore, we calculated the area under the receiver-operating characteristic curve (AUROC) of each model with a 95\% confidence interval (CI). We compared the AUROCs of the above-mentioned two models, calculated the net reclassification improvement (NRI) and integrated discrimination improvement (IDI) to assess the improvement of the predictive value after including the percentage band count into a baseline model. The $p$-values for statistical tests were two-tailed, and a $p$-value $<0.05$ was considered statistically significant. All statistical analyses were performed using R version 3.6.1 (The R Foundation for Statistical Computing, Vienna, Austria).

\section{Results}

In total, 4879 cases during the study period were considered eligible and 970 cases (inpatient, 559 cases; outpatient, 411 cases) were included in the final analysis. The median age was 73 (range, 63-80) years; 629 (64.8\%) were men; median body temperature was 38.1 (range, 37.2-38.6) ${ }^{\circ} \mathrm{C}$; median white blood cell (WBC) count was 10,600 (range, 7800-11,790) cells $/ \mathrm{mm}^{3}$; median eosinophil count was 20 (range, 0-93) cells $/ \mathrm{mm}^{3}$; and the median percentage band count was $0 \%$ (range, $0-2 \%$ ) (Table 1 ). Overall, bandemia was detected in 151 cases (15.6\%) and bacteremia in 188 cases (19.4\%). Among the 188 cases with bacteremia, 190 micro-organisms were isolated (Table 2). The incidence of bandemia was higher in inpatients than in outpatients (inpatients, $18.4 \%$; outpatients, $11.7 \%$; $p=0.004$ ), while there was no significant differences of the incidence of bacteremia between inpatients and outpatients (inpatients, $19.0 \%$; outpatients, $20.0 \%$; $p=0.700$ ).

Table 1. Characteristics of the distinguished groups (bacteremia and no bacteremia).

\begin{tabular}{|c|c|c|c|}
\hline & Bacteremia $(\mathrm{N}=188)$ & No Bacteremia $(\mathrm{N}=782)$ & $p$-Value \\
\hline Age > $65(\%)$ & $143 / 188(76.1 \%)$ & $530 / 782(67.8 \%)$ & 0.027 \\
\hline Men (\%) & $110 / 188(58.5 \%)$ & $519 / 782(66.4 \%)$ & 0.043 \\
\hline $\begin{array}{l}\text { Body temperature * } \\
>38.0 \text { or }<36.0(\%)\end{array}$ & $135 / 180(75.0 \%)$ & $368 / 735(50.1 \%)$ & 0.001 \\
\hline $\begin{array}{l}\text { White blood cell count } \\
>12,000 \text { or }<4000 \text { cells } / \mathrm{mm}^{3}(\%)\end{array}$ & $100 / 188(53.2 \%)$ & $313 / 782(40.0 \%)$ & $<0.001$ \\
\hline $\begin{array}{c}\text { Eosinophil count } \\
<25 \text { cells } / \mathrm{mm}^{3}(\%)\end{array}$ & $135 / 188(71.8 \%)$ & $379 / 782(48.5 \%)$ & $<0.001$ \\
\hline Bandemia (\%) & $79 / 188(42.0 \%)$ & $72 / 782(9.2 \%)$ & $<0.001$ \\
\hline
\end{tabular}

The unadjusted incidence of bacteremia was significantly higher among patients with bandemia than in those without bandemia (52.3\% vs. $13.3 \%$; OR, 7.15; 95\% CI, 4.91-10.5; $p<0.001)$. The sensitivity and specificity of bandemia for predicting bacteremia were 0.42 (95\% CI, 0.35-0.49) and 0.91 (95\% CI, 0.89-0.93), respectively. This was consistent in outpatients (52.1\% vs. $15.7 \%$; OR, 5.80; 95\%CI, 2.94-11.53; $p<0.001$ ) and inpatients (52.4\% vs. $11.4 \%$; OR, 8.51; 95\% CI, 5.12-14.26; $p<0.001)$. The bandemia was retained as 
an independent predictive factor in a multivariable logistic regression model (OR, 6.13; 95\% CI, 4.02 to $9.40 ; p<0.001$; Table 3).

Table 2. Micro-organisms isolated in bacteremia.

\begin{tabular}{ll}
\hline Microbiology & Isolate Number $\mathbf{N}=\mathbf{1 9 0})$ \\
\hline Escherichia coli & $52(27.4 \%)$ \\
Staphylococcus aureus & $37(19.5 \%)$ \\
Klebsiella pneumonia & $26(13.7 \%)$ \\
Klebsiella oxytoca & $9(4.7 \%)$ \\
Enterococcus faecalis & $8(4.2 \%)$ \\
Coagulase-negative staphylococcus & $7(3.7 \%)$ \\
Enterobacter cloacae & $6(3.2 \%)$ \\
Pseudomonas aeruginosa & $6(3.2 \%)$ \\
Streptcoccus pneumonia & $6(3.2 \%)$ \\
Streptcoccus agalactiae & $4(2.1 \%)$ \\
Bacteroides spp. & $4(2.1 \%)$ \\
Serratia marcescens & $3(1.6 \%)$ \\
Group G streptococci & $3(1.6 \%)$ \\
Other Streptococcus spp. & $6(3.2 \%)$ \\
Miscellaneous & $13(6.8 \%)$ \\
\hline
\end{tabular}

Table 3. Odds ratios of variables for predicting bacteremia.

\begin{tabular}{|c|c|c|c|c|}
\hline & $\begin{array}{c}\text { Odds Ratio } \\
\text { (Univariate Model) }\end{array}$ & $p$-Value & $\begin{array}{c}\text { Odds Ratio } \\
\text { (Multivariate Model) }\end{array}$ & $p$-Value \\
\hline Bandemia & 7.15 (4.91-10.50) & $<0.001$ & $6.13(4.02-9.40)$ & $<0.001$ \\
\hline Age $>65$ & $1.51(1.05-2.20)$ & 0.028 & $1.46(0.97-2.22)$ & 0.075 \\
\hline Male & $0.71(0.52-0.99)$ & 0.043 & $0.86(0.59-1.24)$ & 0.411 \\
\hline $\begin{array}{l}\text { Body temperature } \\
>38.0 \text { or }<36.0\end{array}$ & $2.99(2.09-4.36)$ & $<0.001$ & $3.22(2.18-4.84)$ & $<0.01$ \\
\hline $\begin{array}{c}\text { White blood cell count } \\
>12,000 \text { or }<4000 \text { cells } / \mathrm{mm}^{3}\end{array}$ & $1.70(1.24-2.35)$ & $<0.001$ & $1.15(0.79-1.66)$ & 0.471 \\
\hline $\begin{array}{l}\text { Eosinophil count } \\
<25 \text { cells } / \mathrm{mm}^{3}\end{array}$ & $2.71(1.92-3.86)$ & $<0.001$ & 1.99 (1.35-2.97) & 0.001 \\
\hline
\end{tabular}

Data are shown as odds ratio (95\% confidence interval). Odds ratios and $p$-values are derived from logistic regression models.

Furthermore, the AUROC of the prediction model for bacteremia was statistically improved from 0.71 (95\% CI 0.67-0.75) to 0.77 (95\% CI 0.73-0.80) in the band-count model compared to the baseline model (Figure 1). Similarly, the NRI $(0.62$; 95\% CI 0.47-0.76; $p<0.01)$ and IDI $(0.09 ; 95 \%$ CI $0.07-0.12 ; p<0.01)$ were statistically significant. 


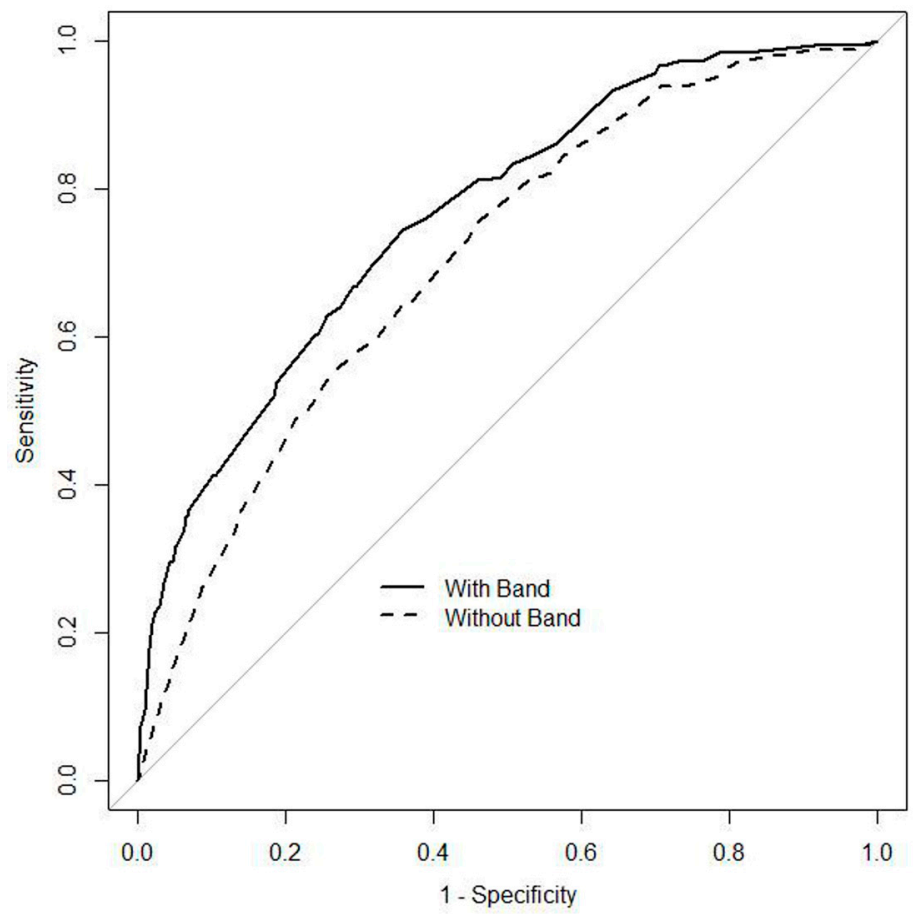

Figure 1. Receiver operating characteristic curves of multivariable logistic regression models for predicting bacteremia. The 'With Band' curve includes age, sex, body temperature, WBC count, eosinophil count, and band count percentage, while the 'Without Band' curve includes age, sex, body temperature, WBC count, and eosinophil count.

\section{Discussion}

This study presents three main findings. First, in patients considered as having indicators for blood cultures by a physician, the prevalence of bacteremia was significantly higher among patient with than without bandemia. Second, bandemia revealed a high specificity and OR for predicting bacteremia. Third, the band-count percentage as a continuous variable, was an independent predictor for bacteremia, and the AUROC was improved by adding the band-count percentage as a continuous variable to the baseline predictive model including age, sex, body temperature, WBC count, and eosinophil count. Our findings support the value of bandemia for predicting bacteremia among patients with a suspected bacterial or fungal infection.

Consistent data from recent studies indicate bandemia (band count $>5 \%$ or $10 \%$ ) as an independent predictor of bacteremia in patients who presented to the emergency department $[17,28]$ and were admitted to general wards [26] or to an intensive care unit [29]. Our study confirms the value of bandemia for the prediction of bacteremia in a more extended population of patients who presented to outpatient department or inpatients who developed a fever after admission. Additionally, our study suggests that the predictive value of bandemia seems to be independent of the total white blood cell count. Even after adjustment and including other factors, such as the WBC count, the OR of band count percentage for predicting bacteremia was stable; previous study results corroborate this finding $[16,26]$. In a retrospective study that included only patients with a normal WBC count on admission, bandemia (band count $>10 \%$ ) was a strong predictor of bacteremia [16]. Another prospective cohort study also showed that bandemia (band count $>5 \%$ ) was observed in $79 \%$ of patients with bacteremia who had a normal WBC count [28]. In addition, the band-count percentage was not associated with the total WBC count in patients with sepsis [29]. Moreover, the study reported that the band count percentage, rather than the WBC count, could distinguish patients with sepsis from those with non-infectious systemic inflammatory response syndrome [29]. Therefore, bandemia could be a useful marker for predicting bacteremia irrespective of the WBC count. 
The strengths of this study include its demonstration of bandemia as a risk factor for bacteremia in both inpatient and outpatient settings and the use of machine-determined bandemia techniques, leading to an easy and objective determination of results. The advantage of bandemia is that the presence or absence of findings can be determined inexpensively, using labor-saving and labor-free machine counts. In recent years, new techniques such as an antibody microarray coupled to a Surface Plasmon Resonance imager (SPRi), PCR methods and Fluorescent in situ hybridization (FISH), were developed [32-35]. These techniques are labor-intensive and costly at the moment; future developments may advance diagnostic strategies for bacteremia. Additionally, there is a proportional relationship between the extent of bandemia and the risk of bacteremia. In addition, 52.3\% of the patients in the bandemia group had bacteremia, and the presence of bandemia led to the prediction of bacteremia with a sensitivity and specificity of 0.42 and 0.91 , respectively. Studies on clinical prediction rules for bacteremia are characterized by high sensitivity and low specificity because they focus on the presence of bacteremia $[17,19,20]$. The prediction of blood cultures by machine learning using Neural Networks has a specificity and sensitivity of $88 \%$ and $17 \%$, respectively [24]. Therefore, if E-alert (which recommends the collection of blood cultures based on a diagnosis od bandemia) is incorporated, it could be well-received by clinicians because of its high specificity and possible assistance in the timely diagnosis of bacteremia.

There are several limitations to this study which should be acknowledged in the interpretation of the results for practice. Th most important limitations are the retrospective study design, the lack of specific criteria indicating the collection of blood cultures (selection bias could, therefore, not be avoided), and the non-inclusion of other objective variables in the analysis, such as vital signs, owing to the lack of data. Second, the conditions under which CBCs were collected may not be constant. Whether the patient is fasting or not, the time of collection, and the location of the blood collection were not included in this analysis. Third, this is not a prospective study of CDSS, although we have shown that bandemia is a useful trigger for noticing bacteremia in both in- and out-patients. Fourth, this study excluded children and, therefore, may not be applicable to pediatric populations. Lastly, our data were derived from a single tertiary academic hospital. As such, the study population tended to present serious medical conditions which, arguably, could be the reason for the high incidence of bacteremia. Additionally, several patients were omitted based on the exclusion criteria. Therefore, the study results should be applied with caution in patients who present with at least one of the exclusion criteria. The next step is to validate our results in a prospective CDSS study that incorporates the exclusion criteria.

\section{Conclusions}

In conclusion, this study demonstrated that bandemia is a useful predictor for bacteremia, regardless of the care setting, and that there is a correlation between bandemia and an increased risk of bacteremia in adults. Therefore, the inclusion of bandemiabased electronic alerts or CDSS for blood-culture collection may improve the diagnosis of bacteremia in both outpatient and inpatient settings in adults.

Author Contributions: T.H. (Taku Harada) and T.S. designed the study. T.H. (Taku Harada) K.M. and T.H. (Takanobu Hirosawa) collected the data. T.H. (Taku Harada) and Y.H. analyzed the data. T.H. (Taku Harada) and Y.H. wrote the manuscript and K.M., T.H. (Takanobu Hirosawa) and T.S. revised it. All authors have read and agreed to the published version of the manuscript.

Funding: This study received no external funding.

Institutional Review Board Statement: This study was approved by the Ethics Committee of Dokkyo Medical University Hospital and conducted in accordance with the Declaration of Helsinki.

Informed Consent Statement: The need for written informed consent was waived because of the retrospective study design. 
Data Availability Statement: The datasets generated and/or analyzed during the current study are not publicly available as the ethics committee did not provide permission, but they are available from the corresponding author upon reasonable request.

Conflicts of Interest: The authors declare no conflict of interests.

\section{References}

1. Coburn, B.; Morris, A.M.; Tomlinson, G.; Detsky, A.S. Does this adult patient with suspected bacteremia require blood cultures? JAMA 2012, 308, 502-511. [CrossRef] [PubMed]

2. Leibovici, L.; Samra, Z.; Konigsberger, H.; Drucker, M.; Ashkenazi, S.; Pitlik, S.D. Long-term survival following bacteremia or fungemia. JAMA 1995, 274, 807-812. [CrossRef] [PubMed]

3. Goto, M.; Al-Hasan, M.N. Overall burden of bloodstream infection and nosocomial bloodstream infection in North America and Europe. Clin. Microbiol. Infect. 2013, 19, 501-509. [CrossRef] [PubMed]

4. Rodríguez-Baño, J.; López-Prieto, M.D.; Portillo, M.M.; Retamar, P.; Natera, C.; Nuño, E.; Herrero, M.; del Arco, A.; Muñoz, A.; SAEI/SAMPAC Bacteraemia Group; et al. Epidemiology and clinical features of community-acquired, healthcare-associated and nosocomial bloodstream infections in tertiary-care and community hospitals. Clin. Microbiol. Infect. 2010, 16, 1408-1413. [CrossRef]

5. Søgaard, M.; Nørgaard, M.; Dethlefsen, C.; Schønheyder, H.C. Temporal Changes in the Incidence and 30-Day Mortality associated with bacteremia in hospitalized patients from 1992 through 2006: A population-based cohort study. Clin. Infect. Dis. 2011, 52, 61-69. [CrossRef]

6. Lenz, R.; Leal, J.R.; Church, D.L.; Gregson, D.B.; Ross, T.; Laupland, K.B. The distinct category of healthcare associated bloodstream infections. BMC Infect. Dis 2012, 12, 85. [CrossRef]

7. Holmbom, M.; Giske, C.G.; Fredrikson, M.; Östholm Balkhed, Å.Ö.; Claesson, C.; Nilsson, L.E.; Hoffmann, M.; Hanberger, H. 14-Year survey in a Swedish County reveals a pronounced increase in bloodstream infections (BSI). Comorbidity-An independent risk factor for both BSI and mortality. PLoS ONE 2016, 11, e0166527. [CrossRef]

8. Leibovici, L.; Greenshtain, S.; Cohen, O.; Mor, F.; Wysenbeek, A.J. Bacteremia in febrile patients. A clinical model for diagnosis. Arch. Intern. Med. 1991, 151, 1801-1806. [CrossRef]

9. Van Dissel, J.T.; Schijf, V.; Vogtländer, N.; Hoogendoorn, M.; van't Wout, J. Implications of chills. Lancet 1998, 352, 374. [CrossRef]

10. Stryjewski, M.E.; Kanafani, Z.A.; Chu, V.H.; Pappas, P.A.; Harding, T.; Drew, L.A.; Benjamin, D.K., Jr.; Reller, L.B.; Lee, B.A.; Corey, G.R.; et al. Staphylococcus aureus bacteremia among patients with health care-associated fever. Am. J. Med. 2009, 122, 281-289.e2. [CrossRef]

11. Kim, K.S.; Kim, K.; Jo, Y.H.; Kim, T.Y.; Lee, J.H.; Lee, S.J.; Rhee, J.E.; Suh, G.J. A simple model to predict bacteremia in women with acute pyelonephritis. J. Infect. 2011, 63, 124-130. [CrossRef]

12. Tokuda, Y.; Miyasato, H.; Stein, G.H.; Kishaba, T. The degree of chills for risk of bacteremia in acute febrile illness. Am. J. Med. 2005, 118, 1417. [CrossRef]

13. Taniguchi, T.; Tsuha, S.; Takayama, Y.; Shiiki, S. Shaking chills and high body temperature predict bacteremia especially among elderly patients. Springerplus 2013, 2, 624. [CrossRef]

14. Komatsu, T.; Onda, T.; Murayama, G.; Yamanouchi, M.; Inukai, M.; Sakai, A.; Kikuta, M.; Branch, J.; Aoki, M.; Tierney, L.M., Jr.; et al. Predicting bacteremia based on nurse-assessed food consumption at the time of blood culture. J. Hosp. Med. 2012, 7, 702-705. [CrossRef]

15. Komatsu, T.; Takahashi, E.; Mishima, K.; Toyoda, T.; Saitoh, F.; Yasuda, A.; Matsuoka, J.; Sugita, M.; Branch, J.; Aoki, M.; et al. A simple algorithm for predicting bacteremia using food consumption and shaking chills: A prospective observational study. $J$. Hosp. Med. 2017, 12, 510-515. [CrossRef]

16. Chase, M.; Klasco, R.S.; Joyce, N.R.; Donnino, M.W.; Wolfe, R.E.; Shapiro, N.I. Predictors of bacteremia in emergency department patients with suspected infection. Am. J. Emerg. Med. 2012, 30, 1691-1697. [CrossRef]

17. Takeshima, T.; Yamamoto, Y.; Noguchi, Y.; Maki, N.; Gibo, K.; Tsugihashi, Y.; Doi, A.; Fukuma, S.; Yamazaki, S.; Kajii, E.; et al. Identifying patients with bacteremia in community-hospital emergency rooms: A retrospective cohort study. PLoS ONE 2016, 11, e0148078. [CrossRef]

18. Lee, C.C.; Wu, C.J.; Chi, C.H.; Lee, N.Y.; Chen, P.L.; Lee, H.C.; Chang, C.M.; Ko, N.Y.; Ko, W.C. Prediction of community-onset bacteremia among febrile adults visiting an emergency department: Rigor matters. Diagn. Microbiol. Infect. Dis. 2012, 73, 168-173. [CrossRef]

19. Shapiro, N.I.; Wolfe, R.E.; Wright, S.B.; Moore, R.; Bates, D.W. Who Needs a Blood Culture? A Prospectively Derived and Validated Prediction Rule. J. Emerg. Med. 2008, 35, 255-264. [CrossRef]

20. Jessen, M.K.; Mackenhauer, J.; Hvass, A.M.S.W.; Ellermann-Eriksen, S.; Skibsted, S.; Kirkegaard, H.; Schønheyder, H.C.; Shapiro, N.I.; CONSIDER Sepsis Network. Prediction of bacteremia in the emergency bepartment: An external validation of a clinical decision rule. Eur. J. Emerg. Med. 2016, 23, 44-49. [CrossRef]

21. Poses, R.M.; Anthony, M. Availability, wishful thinking, and physicians' diagnostic judgments for patients with suspected bacteremia. Med. Decis. Mak. 1991, 11, 159-168. [CrossRef] [PubMed] 
22. Harada, T.; Miyagami, T.; Kunitomo, K.; Shimizu, T. Clinical decision support systems for diagnosis in primary care: A scoping review. Int. J. Environ. Res. Public Health 2021, 18, 8435. [CrossRef] [PubMed]

23. Paul, M.; Andreassen, S.; Nielsen, A.D.; Tacconelli, E.; Almanasreh, N.; Fraser, A.; Yahav, D.; Ram, R.; Leibovici, L.; TREAT Study Group. Prediction of bacteremia using TREAT, a computerized Decision-Support System. Clin. Infect. Dis. 2006, 42, 1274-1282. [CrossRef] [PubMed]

24. Mahmoud, E.; Al Dhoayan, M.; Bosaeed, M.; Al Johani, S.; Arabi, Y.M. Developing machine-learning prediction algorithm for bacteremia in admitted patients. Infect. Drug Resist. 2021, 14, 757-765. [CrossRef]

25. Bone, R.C.; Balk, R.A.; Cerra, F.B.; Dellinger, R.P.; Fein, A.M.; Knaus, W.A.; Schein, R.M.; Sibbald, W.J. Definitions for sepsis and organ failure and guidelines for the use of innovative therapies in sepsis. The ACCP/SCCM Consensus Conference Committee. American College of Chest Physicians/Society of Critical Care Medicine. Chest 1992, 101, 1644-1655. [CrossRef]

26. Drees, M.; Kanapathippillai, N.; Zubrow, M.T. Bandemia with normal white blood cell counts associated with infection. Am. J. Med. 2012, 125, 1124.e9-1124.e15. [CrossRef]

27. Fontanarosa, P.B.; Kaeberlein, F.J.; Gerson, L.W.; Thomson, R.B. Difficulty in predicting bacteremia in elderly emergency patients. Ann. Emerg. Med. 1992, 21, 842-848. [CrossRef]

28. Seigel, T.A.; Cocchi, M.N.; Salciccioli, J.; Shapiro, N.I.; Howell, M.; Tang, A.; Donnino, M.W. Inadequacy of temperature and white blood cell count in predicting bacteremia in patients with suspected infection. J. Emerg. Med. 2012, 42, 254-259. [CrossRef]

29. Mare, T.A.; Treacher, D.F.; Shankar-Hari, M.; Beale, R.; Lewis, S.M.; Chambers, D.J.; Brown, K.A. The diagnostic and prognostic significance of monitoring blood levels of immature neutrophils in patients with systemic inflammation. Crit Care 2015, 19, 57. [CrossRef]

30. Doern, G.V.; Carroll, K.C.; Diekema, D.J.; Garey, K.W.; Rupp, M.E.; Weinstein, M.P.; Sexton, D.J. Practical guidance for clinical microbiology laboratories: A comprehensive update on the problem of blood culture contamination and a discussion of methods for addressing the problem. Clin. Microbiol. Rev. 2019, 33, e00009-19. [CrossRef]

31. Hirosawa, T.; Harada, Y.; Morinaga, K.; Takase, H.; Nin, M.; Shimizu, T. Eosinopenia as a diagnostic marker of bloodstream infection in a general internal medicine setting: A cohort study. BMC Infect. Dis. 2020, 20, 85. [CrossRef]

32. Templier, V.; Livacge, T.; Boisset, S.; Maurin, M.; Slimani, S.; Mathey, R.; Roupioz, Y. Biochips for Direct Detection and Identification of Bacteria in Blood Culture-Like Conditions. Sci. Rep. 2017, 7, 9457. [CrossRef]

33. Opota, O.; Jaton, K.; Greub, G. Microbial diagnosis of bloodstream infection: Towards molecular diagnosis directly from blood. Clin. Microbiol. Infect. 2015, 21, 323-331. [CrossRef]

34. Liesenfeld, O.; Lehman, L.; Hunfeld, K.P.; Kost, G. Molecular diagnosis of sepsis: New aspects and recent developments. Eur. J. Microbiol. Immunol. 2014, 4, 1-25. [CrossRef]

35. Źródłowski, T.; Sobońska, J.; Salamon, D.; McFarlane, I.M.; Ziętkiewicz, M.; Gosiewski, T. Classical Microbiological Diagnostics of Bacteremia: Are the negative results really negative? What is the laboratory result telling us about the "gold standard"? Microorganisms 2020, 8, 346. [CrossRef] 